Diabetologe 2020 $16: 551$

https://doi.org/10.1007/s11428-020-00658-z

(C) Springer Medizin Verlag GmbH, ein Teil von Springer Nature 2020

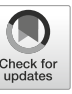

\author{
Matthias Kaltheuner ${ }^{1} \cdot$ Michael Roden ${ }^{2,3,4}$ \\ 'Diabetologikum Leverkusen, Leverkusen, Deutschland \\ ${ }^{2}$ Abteilung für Endokrinologie und Diabetologie, Universitätsklinikum Düsseldorf, Heinrich Heine \\ Universität, Düsseldorf, Deutschland \\ ${ }^{3}$ Institut für Klinische Diabetologie, Deutsches Diabetes-Zentrum (DDZ), Leibniz Zentrum für \\ Diabetesforschung, Düsseldorf, Deutschland \\ ${ }^{4}$ Deutsches Zentrum für Diabetesforschung (DZD e. V.), Neuherberg, Deutschland
}

\section{Lebererkrankungen bei Diabetes mellitus}

tend, da der Verdacht eines übermäßigen Alkoholgenusses bei jedem medizinischen Kontakt nur schwer abwehrbar ist. Die objektive Gefährdung wiederum wird häufig nicht wahrgenommen. So entwickelte sich dem Anschein nach ein therapeutischer Nihilismus, der seinen Ursprung in mangelnden diagnostischen und therapeutischen Möglichkeiten hat.

Mit den Beiträgen in diesem $\mathrm{Heft}$ möchten wir die Bedeutung der Lebererkrankungen bei Diabetes mellitus sowie die diagnostischen und therapeutischen Fortschritte der letzten Jahre aufzeigen.

Roman Liebe und Verena Keitel-Anselmino berichten über die genetischen Hintergründe der Fettlebererkrankung, die die klinische Beobachtung, dass oft keine gute Korrelation von Adipositas und Fettleber besteht, erklären.

Norbert Stefan untersucht die Rolle der Insulinresistenz und des oxidativen Stresses, die Adipositas, Typ-2-Diabetes und NAFLD verbinden.

Jörn Schattenberg und Andreas Geier berichten über neue diagnostische Verfahren bei Diabetes und Fettleber. Nachdem es im Alltag der klinischen Grundversorgung seit Jahrzehnten kaum Veränderungen in der Diagnostik der Fettlebererkrankungen gab, lassen aktuelle Ansätze neue Hoffnung aufkommen.

Jennifer Pützer und Sabine Kahl zeigen den aktuellen Stand der medikamentösen Behandlung der NAFLD bei Diabetes auf. Dabei kann das Wissen des behandelnden Arztes um die Bedeutung der Basistherapie und die Aussicht auf
Besserung durch neue Medikamente die Motivation der Betroffenen steigern.

Das rasch zunehmende Wissen um NAFLD führt dazu, dass in unmittelbarer Zukunft optimierte diagnostische Strategien und die Zulassung neuer Medikamente für diese Erkrankung zu erwarten sind

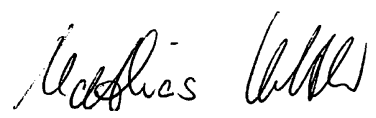

Dr. med. Matthias Kaltheuner

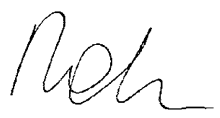

Prof. Dr. med. univ. Michael Roden

\section{Korrespondenzadresse}

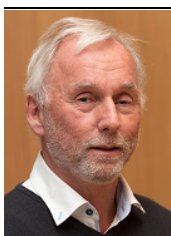

Dr. med.

Matthias Kaltheuner

Diabetologikum Leverkusen

Leverkusen, Deutschland

m.kaltheuner@web.de

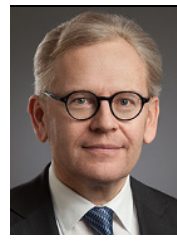

Prof. Dr. med. univ. Michael Roden Institut für Klinische Diabetologie, Deutsches Diabetes-Zentrum (DDZ), Leibniz Zentrum für Diabetesforschung Düsseldorf, Deutschland michael.roden@ddz.de

Interessenkonflikt. M. Kaltheuner und M. Roden geben an, dass kein Interessenkonflikt besteht. erreichen die notwendige Gewichtsabnahme. Für die Patienten ist die chronische leichte Lebererkrankung oft belas- 